\title{
Sentinel Node Biopsy in Breast Cancer: Clinical Implication - Standard of Care - Fututre Prospects
}

\author{
Chair: Anton Haid ${ }^{\mathrm{a}}$ \\ Participants: Thorsten Kühn ${ }^{b}$ Amit Goyal ${ }^{c}$ Christoph Tausch ${ }^{d} \quad$ Florentina Peintinger $^{\mathrm{e}}$ \\ Peter Schrenk $^{f}$ Hans Gallowitsch ${ }^{g}$ \\ ${ }^{a}$ Abteilung für Allgemein- und Thoraxchirurgie, Landeskrankenhaus Feldkirch, Austria \\ ${ }^{\text {b }}$ Frauenklinik, Städtische Kliniken Esslingen a.N., Germany \\ ${ }^{c}$ Department of Surgery, Wales College of Medicine, Cardiff University, UK \\ ${ }^{\mathrm{d}}$ Brustzentrum Zürich, Switzerland \\ ${ }^{\text {e } G y n a ̈ k o l o g i s c h e ~ A b t e i l u n g, ~ L a n d e s k r a n k e n h a u s, ~ L e o b e n, ~}$ \\ ${ }^{f}$ Brustkompetenz-Zentrum, AKH Linz, \\ ${ }^{9}$ Abteilung für Nuklearmedizin und Spezielle Endokrinologie, PET-CT Zentrum am LKH- Klagenfurt, Austria
}

\section{Question 1: Sentinel Node Biopsy Has Been Performed as a Routine Clinical Procedure For the Past Ten Years. What Are Your Personal Experiences with the Procedure and What Is Your Opinion Concerning the Accuracy of this Method for Axillary Staging?}

Kühn: Sentinel node biopsy (SNB) has been accepted as the gold standard for axillary staging in breast cancer. A few years ago we had to justify the indication for SNB. Today we have to justify full axillary dissection as a staging procedure in clinically negative breast cancer patients. This is an important progress for an improved and targeted breast cancer surgery.

Goyal: SNB has been validated in several multicentre studies and is now the standard of care for nodal staging in early breast cancer. The failed localisation rate and the false-negative rate have improved as the technique has evolved. The National Institute of Clinical Excellence (NICE) in the UK has recommended SNB as the preferred technique to stage the axilla. The technique is oncologically safe with no adverse impact on axillary local recurrence and survival.

Tausch: After performing hundreds of SNBs I am still tense at every procedure, even if the majority of these operations work simple. SNB is a fascinating method improving axillary staging in the treatment of breast cancer, which is able to decrease morbidity of the operative procedure. There are no signs for increasing numbers of axillary recurrences by the use of this method.

\section{KARGER}

Fax +497614520714

Information@Karger.de

www.karger.com (c) 2009 S. Karger GmbH, Freiburg

Accessible online at:

www.karger.com/brc
Peintinger: SNB represents a minimally invasive procedure for axillary staging and is reported to be predictive of axillary status. Different mapping agents can be injected: either blue dye alone or in combination with a radioisotope [1]. My personal experience with SNB started in the late 1990s using blue dye alone with a learning period of approximately 50 cases. At this time the identification rate was $96 \%$ and the accuracy was $98.6 \%$ [3]. Currently we are using a radiocolloid in combination with blue dye and the identification rate is slightly higher. In my experience a shorter surgery time is achieved due to easier identification of the sentinel node with dual signalling (visual and acoustical). To evaluate the safety of SNB we observed 200 patients with negative sentinel nodes in a mean follow up of 3 years and found no axillary recurrences [4].

Schrenk: We began using SNB for axillary staging in breast cancer patients in 1996. At that time SNB was rarely used and still considered an experimental procedure. Between 1996 and 1998 we gained further experience with the technique in quite a number of patients, and SNB without axillary lymph node dissection (ALND) in case of a negative sentinel node was used at our clinic since 1998. From 1998 we broadened the indications for SNB and nowadays use it for axillary staging in almost all breast cancer patients. I consider it essential for a surgeon to learn lymphatic mapping and SNB from scratch and with all the tricks. A learning period of a minimum of 30 cases with backup ALND in case of a negative sentinel node and a false-negative rate not higher than $5 \%$ is generally suggested before clinical use. Experience with the procedure is 
required and this cannot be trained watching experienced surgeons doing 5 cases in a weekend workshop. However, most patients request to forego ALND in case of a negative sentinel node and this makes it difficult for surgeons starting with SNB to gain experience with the procedure and do backup controls (through ALND).

Gallowitsch: After the introduction of SNB in Austria, in the beginning there was a lot of scepticism about this minimally invasive procedure as several issues remained unsolved. Due to the activities and dedication of the Austrian Sentinel Node Study Group and all involved disciplines, it soon became clear that this method could only have a chance to be implemented in the therapy of breast cancer patients if high quality standards were met and the indication was clearly defined. The importance of a learning period for surgeons/ gynaecologists, pathologists and nuclear medicine physicians was recognised early and SNB was performed initially only within prospective trials and compared with the results of conventional ALND. The initial relatively high false-negative rate could be reduced to a lower level between 5 and $10 \%$ by multisectional slicing and immunohistochemistry (IHC). In our (Nuclear Medicine) department but also in other departments, different application techniques (peritumoral, subdermal, subareolar or combined), different volumes and amount of injected material were investigated and the procedure could be optimized with currently nearly $100 \%$ detection rates of the sentinel node. This, combined with other advantages was the reason that the surgeons more and more relied on the radiotracer technique in our country, sometimes combined with blue dye. Nevertheless, there remains a certain scepticism regarding to the long-term results which are missing, although the data obtained so far are very promising (see below).

\section{Question 2: In Your Opinion, What Are the Assured Indications and What Are the Areas of Controversy?}

Kühn: All patients who require axillary staging are candidates for SNB. Patients with suspicious axillary nodes should undergo fine needle aspiration (FNA) or core biopsy. The role of SNB in the setting of preoperative chemotherapy (PCT) is unclear.

Goyal: SNB is suitable for all patients with invasive breast cancer and negative nodes in clinical or ultrasound examination, irrespective of tumour size. It may be suitable for selected patients with clinically positive axillae, $25 \%$ of whom have been shown to be node-negative. These patients should undergo ultrasound-guided FNA or core biopsy; node-positive patients can proceed directly to ALND while those who are negative undergo SNB. More data are needed to clarify the role of SNB following neoadjuvant chemotherapy and in patients who develop breast recurrence following breast conservation and SNB.

Tausch: SNB is recommended for T1-2 breast carcinoma patients with clinically negative axilla. It is reliable for unicentric and multicentric tumours. I prefer the method also for highgrade DCIS (ductal carcinoma in situ) with necrosis, especially when the tumour lies in the upper outer quadrant, because if invasion is proved in the definitive histology of the removed specimen the method could be of limited value in a second operation. For non-high-grade DCIS and other tumour sites I do not perform SNB. SNB can be applied in the framework of prophylactic mastectomy for patients with mutation in the BRCA1/2 gene.

Peintinger: The clear indications for SNB are T1-2 invasive breast cancers and a clinically negative axilla. Excisional biopsy and multicentric breast cancer are currently also considered an appropriate indication for SNB [5]. In cases of an extensive DCIS (size $\geq 4 \mathrm{~cm}$, palpable mass, high-grade) requiring mastectomy there is some risk of invasion and SNB is recommended. However, this technique is not recommended for pure DCIS, since the prevalence of (macro)metastatic sentinel nodes is lower than 1\% [6]. In contrast, the clinically positive axilla and inflammatory breast cancer represent contraindications to SNB. For patients receiving PCT it is still controversial whether SNB should be performed before or after chemotherapy. Although there are some advantages for pre-operative SNB (determines need for axillary dissection, successful identification and highly accurate) the disadvantages (second operation, loss of information about nodal response to chemotherapy, potential increase in axillary dissection) outweigh the potential benefit from PCT.

Schrenk: I would like to answer the question the other way around. In my opinion there are only two contraindications to SNB. One is inflammatory beast cancer (very low identification rate, clinically positive node in many patients) and the other is no histologically proved cancer. Indications not generally accepted are multicentric carcinoma, SNB after prior breast or axillary surgery (e.g., local recurrence), T3, T4b cancers and SNB prior to planned preoperative chemotherapy or hormonal treatment. The use of SNB in these patients largely is driven by personal experience and it is just a matter of time before these indications will become standard indications for SNB.

Gallowitsch: Indications are invasive breast cancer histologically proven by biopsy to be stage $\mathrm{T} 1$ or $\mathrm{T} 2(<3 \mathrm{~cm})$, clinically node negative, and large DCIS with invasive component. SNB after PCT and SNB in multicentric cancer have demonstrated reliable results in several studies and will probably become a further assured indication for SNB. 
Fig. 1. Approach at the AKH Linz to sentinel node biopsy if preoperative chemotherapy is planned. Abbreviations: $\mathrm{LN}=$ lymph node, $\mathrm{SN}=$ sentinel node, $\mathrm{ALND}=$ axillary lymph node dissection, $\mathrm{PC}=$ preoperative chemotherapy, FNAB = fine needle aspiration biopsy, $\mathrm{cN} 0=$ clinically node negative, $\mathrm{cN}+=$ clinically node positive.

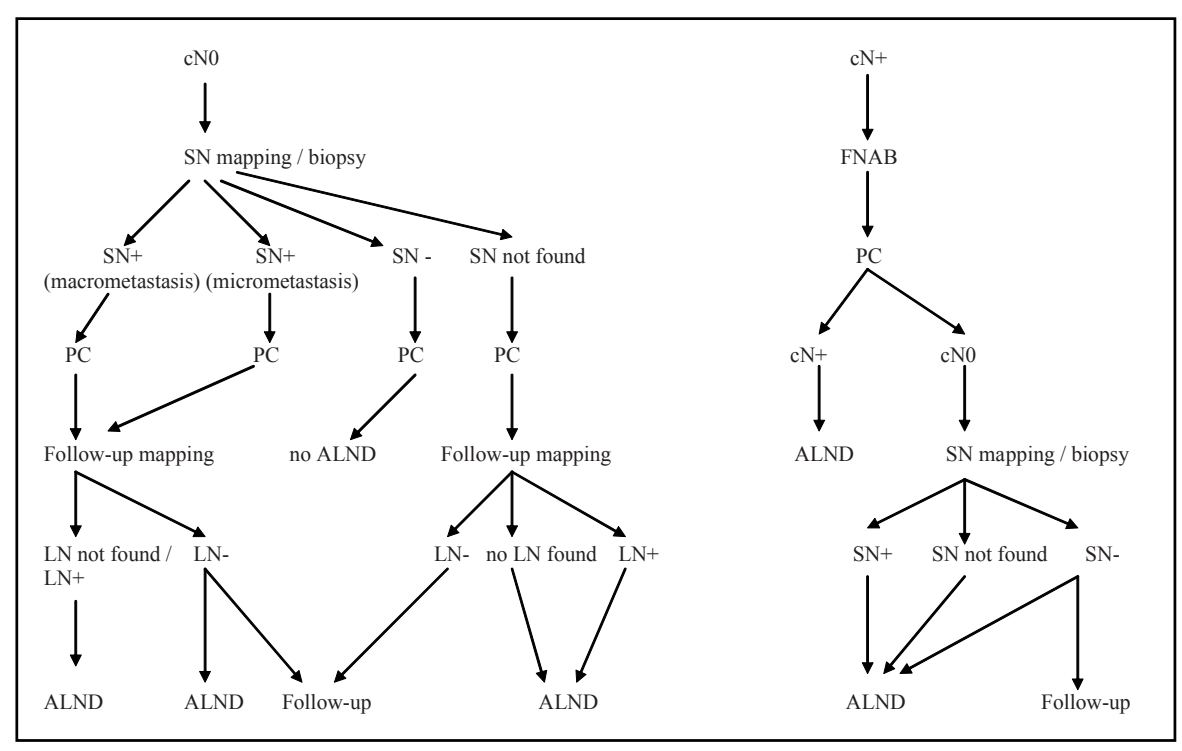

\section{Question 3: How Do You See the Indication of Sentinel Node Biopsy within Preoperative Chemotherapy in Early-Stage Breast Cancer?}

Kühn: There is no consensus on the role of SNB in patients who undergo PST. Since SNB is a staging procedure it might influence local and systemic treatment decisions in nodenegative patients if it was performed prior to chemotherapy. On the other hand, patients who convert from a positive to a negative axillary status could be spared axillary dissection, if SNB was reliable in this setting. The German SENTINA-Trial examines the role of SNB in the neoadjuvant setting.

Goyal: The timing of SNB with respect to neoadjuvant chemotherapy in early stage breast cancer patients remains a subject of debate. Pre-chemotherapy SNB accurately stages the axilla and may affect the choice of chemotherapy regimens used in sentinel node-positive patients. Post-chemotherapy SNB avoids a second surgery and does not delay the start of neoadjuvant chemotherapy. It identifies node-positive patients who have been downstaged to N0 by neoadjuvant chemotherapy and therefore can be spared ALND. However, neoadjuvant therapy may achieve a 'patchy' kill of metastatic disease in the axillary lymph nodes leading to a higher false-negative rate. In Cardiff, we perform SNB before primary systemic therapy, with ALND performed after chemotherapy if disease is present in the sentinel node.

Tausch: After PCT SNB is reliable but is afflicted with a higher false-negative rate and cannot be recommended outside of clinical trials. There is little experience with SNB before PCT which can better predict the axillary status. The benefit of axillary downstaging by PCT is lost and a second operation is necessary. In our institution SNB is performed before PCT combined with the implantation of a port-a-cath system.
Peintinger: To date there are enough data supporting the implementation of SNB in patients receiving PCT. The purpose of SNB in this setting is the appropriate surgical and radiation treatment based on the information about the extent of disease before as well as after chemotherapy. For adequate planning of breast conserving treatment or mastectomy with or without axillary radiation it is essential for patients to be staged accurately. However, the appropriate timing of SNB is still under discussion. It is important to know that SNB after chemotherapy is as feasible and accurate as it is before chemotherapy. In my opinion, pre-SNB is associated with a major disadvantage. Given that PCT converts up to $40 \%$ of positive axillary nodes into negative nodes, a number of patients will be treated unnecessarily with an axillary dissection. Also, the loss of information about the response of the removed sentinel node(s) to chemotherapy may provide false prognostic information.

Schrenk: We routinely perform SNB prior to PCT in patients with a clinically negative axilla. Patients with a clinically (or sonographically) positive axilla undergo FNA biopsy to verify metastatic disease and then are scheduled to have SNB after PCT. The algorithm in figure 1 shows our approach to SNB in case a PCT is planned [7].

Gallowitsch: SNB before PCT can be used to stratify the axillary intervention after PCT. Schrenk et al. [7] demonstrated a $0 \%$ positivity rate after PCT when the sentinel node was negative or had only micrometastatic disease before PCT. Therefore, a negative sentinel node can be a predictor of a negative axilla after PCT and ALND could be abandoned in these patients. SNB after PCT is another issue which should be discussed. The interesting question in this context is: Why should you expect tumour cells in the sentinel node when even the primary tumour shows histological remission after PCT? Is it 
a different behaviour and response to PCT of metastatic tumour cells in the sentinel node and the primary tumour? Is it the diminished accessibility of the metastatic cells in the sentinel node for the PCT? According to the results of the Austrian Sentinel Node Study Group, there was a $42 \%$ positivity rate in the sentinel node after 3-6 courses of chemotherapy which is supported also by Grube et al. [8] (55\% positivity), even if the primary tumour in the breast demonstrated complete histologic response. According to the results of several studies, the results after PCT are quite comparable to the conventional sentinel node procedure and seem to be reliable.

\section{Question 4: What Do You Think about the Use of Axillary Ultrasound with or without FNA/Core Biopsy of Lymph Nodes for Pre-Operative Axillary Staging?}

Kühn: Patients with a clinically positive axillary status are often excluded from SNB; however, $25 \%$ of these patients turn out to be histologically negative. Since full axillary dissection is associated with a high morbidity, the indication should not be based on clinical findings but on histologic results. The rate of patients with a $\mathrm{pN} 0$-status who receive full axillary dissection should be defined as a (negative) quality measure.

Goyal: Axillary ultrasound is a quick, noninvasive outpatient procedure but the results are operator dependent. It should be performed in all patients who are investigated for early invasive breast cancer and, if morphologically abnormal lymph nodes are identified, ultrasound-guided FNA or core biopsy should be performed. Patients with FNA or biopsy proven metastasis can undergo axillary lymph node dissection, thus avoiding unnecessary radiation exposure, morbidity, cost and inconvenience of SNB. All patients with an ultrasound-negative FNA or biopsy of an abnormal node should undergo SNB because of the high false-negative rate. Our own data show that axillary ultrasound can avoid unnecessary SNB in 30\% of node-positive patients.

Tausch: We perform ultrasound of the axilla and in the case of a suspicious lymph node we do FNA to verify the suspicion. This way we can identify a higher rate of node-negative patients preoperatively.

Peintinger: Clinical assessment of axillary nodal status before axillary surgery by ultrasound alone has a low sensitivity in predicting subclinical disease. Overall sensitivity is approximately $60 \%$. A reasonable approach, as seen at the MD Anderson Cancer Center, is the ultrasound-guided FNA in patients with breast cancer. FNA and core biopsy show a higher sensitivity compared to ultrasound ( 75 and $82 \%$, respectively) for predicting axillary status [9]. Although FNA is a cost-effective approach (in contrast to core biopsy) for accurate FNA a high amount of experience in technique and cytology is required.
Schrenk: Axillary staging prior to surgery (using clinical examination and sonography) is mandatory to exclude or identify positive / suspicious lymph nodes (although sonography is associated with a high false-negative rate, which may be as high as $30 \%$ ). In case of suspicious axillary lymph nodes we perform a sonographically guided FNA biopsy to diagnose metastatic disease, which allows us to perform a primary axillary dissection instead of wasting time on SNB. Whenever FNA biopsy shows no metastatic lymph node we do a SNB.

Gallowitsch: Similar to PET and CT, the negative predictive value and accuracy of axillary ultrasound to exclude axillary micrometastases is too low to accurately stage the axilla. The accuracy of PET, ultrasonography and the combination of both revealed an overall accuracy of 82,79 and $85 \%$, respectively [10]. Nevertheless, with an experienced investigator and combined with FNA or core needle biopsy, it is a useful and highly specific tool to exclude lymph node macrometastases which obviates the use of SNB in these patients [9], but substantially increases the costs.

\section{Question 5: Which Kind of Sentinel Node Mapping Do You Prefer in Your Clinical Practice? Does the Combined Method with Blue Dye and Radiocolloids Make Sense?}

Kühn: The use of a radiocolloid is mandatory for us. The additional use of blue dye is optional and may help to identify the sentinel node faster in some cases. We rarely identify additional sentinel nodes with blue dye alone. I do not believe that the combined technique reduces the false-negative rate.

Goyal: Our standard method is to inject the isotope intradermally at a single periareolar site in the index tumour quadrant (or cephalad of the previous biopsy scar), in a volume of $0.05 \mathrm{ml}$ of normal saline. Patent blue $\mathrm{V}$ injection is always given in the operating room just prior to surgery. We inject subdermally at a single periareolar site in the index tumour quadrant. All patients undergo pre-operative lymphoscintigraphy at least 15 minutes after the radioisotope injection. However, we do not think this is necessary once the surgeons are past their learning period. This injection technique has a failed-localisation rate of $1 \%$ and a false-negative rate of less than $10 \%$. We and the NICE guidelines (UK) recommend the use of combined technique to maximize both the success and accuracy of SNB. Our data show that the false-negative rate will increase by $3-4 \%$ by relying on a single technique of localisation.

Tausch: We prefer the radiocolloid tracer and subareolar injection. In more than $95 \%$ the scintigraphy shows a sentinel node in the axilla and we can avoid the time-consuming blue dye injection. 
Peintinger: In clinical routine I prefer the combined lymphatic mapping method for the practical reasons I mentioned before. We perform scintigraphic localisation of the sentinel node(s) by peritumoural injection of radiocolloid (Tc-99 labelledalbumin, Nanocoll ${ }^{\circledR}$ ) 16-18 h before surgery. Intra-operatively we inject $2 \mathrm{ml}$ blue dye (Patent Blue ${ }^{\circledR}$ ) subareolarly. We do not use peritumoural injection of blue dye to avoid discolouration of the tissue. In my opinion the experienced surgeon may offer highly accurate performance with both methods of lymphatic mapping used in combination or alone.

Schrenk: Since we have started out SNB using the vital blue dye for mapping and used it almost exclusively for 2 years we rapidly gained experience with the dye alone. However, whenever possible we prefer the combined approach with blue dye and radiocolloid. This is especially mandatory with indications such as prior surgery to the breast and/or axilla (e.g. reoperative SNB in case of local recurrence), prior or after PCT, multicentric carcinoma, and when we try to avoid a separate incision in the axilla. In these cases the combined approach helps in identification of extra-axillary sentinel node(s) outside the standard lymphatic drainage pattern to the axilla. We inject around the tumour (when using blue dye alone) or around the tumour (blue dye) and subareolarly (radiocolloid). Studies found that the peritumoural injection is comparable to the subareolar injection with respect to identification rate and false-negative rate.

Gallowitsch: Most of my surgical colleagues use both methods in the learning phase to become familiar with the method. After this period many of them use only the radiocolloid method because of its obvious advantages: a long time window with the possibility to perform the application $24 \mathrm{~h}$ prior to the surgery, stable retention in the sentinel node, easy access and localisation with the help of the gamma probe and no tattooing. This is only possible after passing a learning phase for all involved disciplines.

\section{Question 6: Which Method of Intraoperative Histopathologic Examination Is Your Everyday Routine and How Reliable Is It?}

Kühn: Intraoperatively the sentinel node is examined by frozen section in our institution. Although the in literature intraoperative assessment of sentinel nodes is associated with a false-negative rate of $>20 \%$ we have few secondary interventions. These are mainly due to micrometastases.

Goyal: We routinely use the Gene Search ${ }^{\mathrm{TM}}$ BLN (breast lymph node) Assay for intraoperative assessment of the sentinel node. The assay can be performed and the results interpreted by a trained technician without the need of a pathologist. The assay has a sensitivity of over $87 \%$ and a specificity of over $94 \%$. The turnaround time of the assay is comparable to that of frozen section $(\sim 30 \mathrm{~min})$.

Tausch: In our everyday routine the sentinel node is sectioned in 2-mm slices during the operation. These slices are examined by loupe. If suspicious areas appear, a frozen section will be performed of this slice. Our pathologists take care to spare tissue of the lymph node as much as possible for routine $\mathrm{H}$ and E staining and IHC. By this technique sentinel metastases are found intraoperatively in $6.0 \%$ but in $11.5 \%$ the metastasis is only seen in the definitive examination.

Peintinger: Frozen section is the conventional method we use for the intraoperative evaluation of the sentinel lymph node in clinical routine. Unfortunately, there are a number of disadvantages using this method, such as loss of tissue, potential loss of micrometastases, freezing artefacts and fatty axillary lymph nodes that are difficult to cut. The reported sensitivity of frozen section is around $72 \%$ in comparison to touch imprint cytology $(50 \%)$ and rapid cytokeratin immunostaining in combination with frozen section (83\%) [11]. Alternatives may represent frozen section of the entire sentinel node as performed in Milan - a method that is time consuming and cost ineffective. In the near future we plan to use intraoperative assays, which have shown a sensitivity of around $95 \%$ and results available within 30-40 $\mathrm{min}$ [12].

Schrenk: We always do a frozen section of the sentinel node, which avoids a secondary ALND in about $35 \%$ of patients compared to when no frozen section is done. Frozen section consists of bisection of the node and 3 sections of one half. In some patients we do additional intraoperative IHC on these sections. This approach diagnoses almost all patients presenting with sentinel node macrometastases. Contrary to that most micrometastases and isolated tumour cells (ITC) are only found in the permanent sections. Overall around 10\% of our patients with a negative SNB in the frozen section are diagnosed with metastatic disease in the permanent sections. I would prefer a complete intraoperative examination of the sentinel nodes(s) with IHC (as they do it in the European Cancer Institute in Milan) to avoid secondary axillary surgery. However, this is only practicable in large centres but is impossible in everyday routine.

Gallowitsch: In our hospital, frozen section analysis is performed in each patient followed by serial multislice sectioning and ICH according to the Austrian standard defined by the Austrian Society of Pathologists and the Sentinel Node Study Group. According to the literature, frozen section analysis is very sensitive (about 98\% [13]) to detect macrometastases in the sentinel node and delayed completion ALND can be avoided in $98 \%$ of patients who underwent SNB with frozen section analysis. The conversion rate between frozen section and definitive histology (including ICH) in more than 1,160 
patients in the Austrian multicentre study was about 13\% which means that only this proportion had secondary ALND whereas a third of patients without frozen section had to undergo secondary ALND. After passing a learning phase even for the pathologist, multisectional $\mathrm{H}$ and $\mathrm{E}$ staining and IHC seems to be very reliable to accurately diagnose the sentinel node status. The balance between the costs and the workload for the pathologists on one hand and the histopathologic outcome on the other hand is still a matter of debate.

\section{Question 7: Due to Special Examination of Sentinel Nodes Micrometastases Are Found More Often. How Do You Decide in Case of Intraoperative versus Postoperative Finding of Micrometastases Regarding Axillary Dissecton?}

Kühn: Today we only have data on non-sentinel-node involvement in patients with micrometastases in the sentinel node (10-20\%). For this reason, we recommend secondary axillary dissection in these patients. We have, however, insufficient data on the outcome of patients who do not undergo further surgery. I believe that we will abandon full axillary surgery in patients with micrometastases very soon. However, the data are not available yet.

Goyal: We routinely offer completion axillary lymph node dissection to patients with sentinel node micrometastasis as additional $\mathrm{H}$ and $\mathrm{E}$ detectable disease within non-sentinel nodes is found in $15-20 \%$ of these patients.

Tausch: In case of intra-operative finding of metastases a completing ALND is mandatory. If a micrometastasis is only found at postoperative work-up of the sentinel lymph node the decision for secondary axillary dissection is influenced by patient-related factors like age, comorbidity, affinity to a new operation and the special need for safety. Additionally we can enrol our patients with micrometastases to an open clinical trial (IBCSG-23).

Peintinger: The standard of care is to perform axillary clearance in case of micrometastatic disease in the sentinel lymph node. However, the prognostic significance of micrometastases is still uncertain: the incidence of non-sentinel metastases is between 13 and $22 \%$ and of those only $4 \%$ will have an axillary recurrence (total of $0.8 \%$ ). Based on this information axillary dissection can be omitted in selected patients. In case of favourable patient and tumour characteristics (small tumour size, highly differentiated, endocrine responsive tumour and elderly patient with co-morbidities) we abandon axillary clearance when micrometastatic disease was found in the sentinel node intra- or postoperatively. In these patients postoperative treatment decisions would not change significantly if axillary clearance was performed. In contrast, axillary morbidity would decrease quality of life with no clear benefit for overall survival.

Schrenk: When the frozen section reveals a metastatic sentinel node (macro- or micrometastasis) we perform ALND of the first and second level. In case of a negative sentinel node in the frozen section but macrometastatic sentinel node in the permanent examination we always do a secondary ALND. In case of micrometastases found in the permanent examination we suggest ALND when the metastatic size exceeds $1 \mathrm{~mm}$. In case of micrometastases $<1 \mathrm{~mm}$ or ITC we inform the patient that there is a small (5-14\%) chance of further positive nodes in the axilla, and do not suggest further ALND. This approach, however, requires informed consent of the patients and all patients are followed up in clinical studies using a clinical and sonographic axillary examination every 3 months [14].

Gallowitsch: No comment.

\section{Question 8: Wherein Do You See the Greatest Advantages of the Sentinel Node Concept?}

Kühn: SNB is a precise staging procedure for the $\mathrm{pN}$ status. It reduces shoulder-arm morbidity significantly. The quality of life of patients with early breast cancer is improved.

Goyal: Molecular analysis of the sentinel node using real-time RT PCR-based assays is the greatest advance in SNB. However, it raises a number of interesting issues. Should a sample of the lymph node be paraffin embedded and archived or should the whole node be submitted for the PCR assay to minimise sampling error? The current recommendation is that the sentinel nodes should be handled as in the validation studies, i.e. sliced fresh into $1.5-3-\mathrm{mm}$ slabs and alternate slabs should be submitted for PCR and paraffin section histopathology. The node homogenates cannot be stored indefinitely and this approach allows permanent archival material to be stored in paraffin wax.

Tausch: The greatest advantages are the decreasing axillary morbidity for half of our patients and the better understanding of lymph node involvement of the axillary basin since we perform SNB.

Peintinger: SNB is a diagnostic test predicting the axillary status accurately and is associated with a low morbidity and a better quality of life.

Schrenk: A negative SNB does not require an ALND and is associated with negligible morbidity. This is best seen when a positive sentinel node in the permanent examination requires secondary ALND. The more exact examination of the sentinel node(s) allows diagnosis of more patients with metastatic 
lymph nodes, but with the impact of a micrometastatic sentinel node on further outcome is still discussed controversially. One major benefit of sentinel node mapping is the information we gain regarding lymphatic drainage patterns of the breast. This is of clinical importance in patients with lymphatic drainage patterns exclusively to the internal mammary artery nodes or in case of an extra-axillary as well as an axillary drainage. Axillary dissection only in these cases would carry the risk of operating on the wrong lymphatic basin.

Gallowitsch: The greatest benefit is the diminished ALNDassociated morbidity regarding lymph oedema, plexus paresis and pain following ALND which can be avoided by the SNB procedure. Furthermore, axillary staging has been improved, especially by a more accurate detection of micrometastases by multisectional slicing and immunohistochemical staining.

\section{Question 9: Do You Have Ideas to Improve the Sentinel Node Concept?}

Kühn: The technique of SNB appears well established and is highly reproducible. We can discuss the necessity of lymphoscintigraphy in order to reduce costs. Numerous (mainly oncologic) questions related to the role of micrometastases, extra-axillary lymph nodes or the need for full axillary dissection in $\mathrm{pSLN}+$ patients require further investigation.

Goyal: The next step is to develop an ultrasound-based alternative to radioisotope and blue dye injection to identify the sentinel node. We need to develop safe and effective contrast agents for ultrasound imaging that will travel to the sentinel nodes when injected in the breast. The sentinel nodes can then be visualised and biopsied in the clinic using ultrasound.

Tausch: Preselection by ultrasound examination of the axilla before SNB and verifying results by FNA can reduce falsenegative results. The establishment of more accurate pathological examination like rapid cytokeratin immunostaining or molecular RT PCR during surgery in order to decrease the rate of secondary axillary procedures.

Peintinger: Further research is needed to understand the biology of metastases in the sentinel node based on molecular assays in order to avoid surgical and/or systemic overtreatment.

Schrenk: No or incomplete data are available on which patients may be spared complete ALND in case of a sentinel node macro- or micrometastasis. This needs further evaluation or validation of the currently suggested models. One big improvement would be to have the final histological result at hand during surgery in order to avoid a secondary ALND. The major task for the future will be to have standard protocols for SNB mapping, pathological examination and follow- up which would rather simplify comparison of the data of different investigators.

Gallowitsch: After passing the learning phase in each involved discipline, quality has now reached a high level with few issues to improve. Pathologists sometimes complain about a lot of sentinel nodes delivered by the surgeons leading to a high workload and also increasing the costs of the procedure. The problem is that both blue dye and radiocolloid pass the sentinel node and mark also secondary echelon lymph nodes which are taken for sentinel nodes by the surgeon. From the diagnostic point of view, a more exact targeting of the sentinel node can be reached by an optimal radiocolloid particle size (delivered by industry) or by optimizing imaging of the sentinel node. As an example, the sentinel node can be discriminated from second echelon lymph nodes in most cases using preoperative SPECT-CT for exact localisation [15].

\section{Question 10: How Do You Assess Personally and as the Result of Literature the Onclogic Saftey of Sentinel Node Biopsy?}

Kühn: All patients treated in our institution undergo a systematic follow-up. Recurrence rates are assessed routinely. Personally, I did not observe a single axillary recurrence after SNB in a 10 -year period.

Goyal: SNB is oncologically safe and this is supported by the results of numerous prospective studies published in the literature. However, we recommend that surgeons offering this procedure should follow up their patients and audit their results.

Tausch: We are still waiting for the results oft the large trials which compare the rate of axillary recurrence between SNB and axillary dissection. By the excessive pathological work-up of the sentinel node we see a larger number of node-positive cancers than we did before the sentinel era.

Peintinger: Veronesi and colleagues [16] found in a recent analysis of a large series of patients with a negative sentinel node that axillary metastases occurred in $0.9 \%$ of the patients and overall survival was $98 \%$ in a median follow-up of 48 months. Overall, the high sensitivity of SNB and the thorough examination of the sentinel node seem to be associated with reduction of residual tumour burden.

Schrenk: The oncological safety of the sentinel node concept has been shown in several studies. They all found a low axillary recurrence rate and when doing a backup ALND a low false-negative rate. However, most of the patients in these studies were operated by surgeons with a vast experience in SNB. What we really need to know with the worldwide spread 
of the sentinel node concept is whether these results are also possible with the vast majority of surgeons who perform SNB in a limited number of patients. The next step, however, will be to examine which patients with micrometastases, ITC and even macrometastases can be spared ALND.

Gallowitsch: Provided that quality standards are met and learning periods have been passed, the oncological safety seems to be at a high level. According to the published results of Konstantiniuk and colleagues [17] of the Austrian Sentinel Node Study Group covering a mean follow-up interval of 34 months after SNB, the results regarding to disease-free survival, local recurrence rate, distant recurrence rates and overall survival are similar to conventional ALND. In a series of 3,548 patients with negative sentinel node followed up for 48 months, Veronesi et al. [16] found only 31 cases with overt axillary metastases $(0.9 \%)$ - which is lower than expected and a 5-year overall survival rate of $98 \%$. Nevertheless, there remains some uncertainty concerning the long-time results.

\section{Participants}

Prof. Dr. med. Thorsten Kühn

Frauenklinik und Interdisziplinäres Mammazentrum Esslingen Hirschlandstr. 97, 73730 Esslingen, Germany

Tel. +49 711 3103-3051, Fax -3052

T.Kuehn@klinikum-esslingen.de

\section{Amit Goyal MS, DNB, FRCS}

Department of Surgery, Wales College of Medicine, Cardiff University, Cardiff, CF14 4XN, United Kingdom

Tel. +442920742758

GoyalA@cf.ac.uk

Dr. med. Christoph Tausch

Brust-Zentrum Zürich

Seefeldstrasse 214

8008 Zürich, Switzerland

PD Dr. med. Florentina Peintinger

Gynäkologische Abteilung

Landeskrankenhaus

Vordernberger Strasse 42

8700 Leoben, Austria

Univ.-Doz. Dr. med. Peter Schrenk

Brustkompetenz-Zentrum

AKH Linz

Krankenhausstrasse 9

4020 Linz, Austria

Univ.-Doz. Dr. med. Hans-Jürgen Gallowitsch

Abteilung für Nuklearmedizin und Spezielle Endokrinologie

PET-CT Zentrum am LKH Klagenfurt

St. Veiterstrasse 47, 9020 Klagenfurt, Austria

hans-juergen.gallowitsch@lkh-klu.at

\section{References}

1 Giuliano AE, Kirgan DM, Guenther JM, Morton DL: Lymphatic mapping and sentinel lymphadenectomy for breast cancer. Ann Surg1994;220:391-398.

$\checkmark 2$ Kuerer HM, Newman LA: Lymphatic mapping and sentinel node biopsy for breast cancer: developments and resolving controversies. J Clin Oncol 2005;23: 1698-1705.

-3 Peintinger F, Reitsamer R, Ralph G. Implementation of sentinel lymph node biopsy with blue dye outside a spesialized center: can we improve quality assurance? Breast 2005;11:103-107.

-4 Reitsamer R, Peintinger F, Prokop E, Rettenbacher L, Menzel C: 200 sentinel node biopsies without axillary lymph node dissection - no axillary recurrences after a 3-year follow-up. Br J Cancer 2004;90:1551-1554.

5 Lyman G, Giuliano A, Veronesi U, et al.: American Society of Clinical Oncology guideline recommendations for SLNB in early-stage breast cancer. J Clin Oncol 2005;23:7703-7720.

6 Intra M, Rotmensz N, Veronesi P, Colleoni M, Iodice $\mathrm{S}$, et al.: Sentinel node biopsy is not a standard procedure in ductal carcinoma in situ of the breast: the experience of the European Institute of Oncology in 854 patients in 10 years. Ann Surg 2008;247:315-319.

7 Schrenk P, Tausch C, Wölfl S, Bogner S, Fridrik M, Wayand W: Sentinel node mapping performed before preoperative chemotherapy may avoid axillary dissection in breast cancer patients with negative or micrometastatic sentinel nodes. Am J Surg 2008;196:176-183.
8 Grube BJ, Christy CJ, Black D, Martel M, Harris L, Weidhaas J, Digiovanna MP, Chung G, Abu-Khalaf MM, Miller KD, Higgins SA, Philpotts L, Tavassoli FA, Lannin DR: Breast sentinel lymph node dissection before preoperative chemotherapy. Arch Surg 2008;143(7):692-699; discussion 699-700.

$\checkmark$ Rao L, Lilley L, Andrews V, Radford L, Ulissey M: Axillary staging by percutaneous biopsy: sensitivity of fine-needle aspiration versus core needle biopsy. Ann Surg Oncol 2009;16:1170-1175.

10 Ohta M, Tokuda Y, Saitoh Y, Suzuki Y, Okumura A, Kubota M, Makuuchi H, Tajima T, Yasuda S, Shohtsu A: Comparative efficacy of positron emission tomography and ultrasonography in preoperative evaluation of axillary lymph node metastases in breast cancer. Breast Cancer 2000;7:99-103.

11 Krishnamurthy S, Meric-Bernstam F, Lucci A, Hwang RF, Kuerer HM, et al.: A prospective study comparing touch imprint cytology, frozen section analysis, and rapid cytokeratin immunostaining for intraoperative evaluation of axillary sentinel lymph nodes in breast cancer. Cancer 2009;115:1555-1562.

12 Visser M, Jiwa M, Horstman A, Brink AA, Pol RP, van Diest $P$, et al.: Intra-operative rapid diagnostic method based on CK19mRNA expression for the detection of lymph node metastases in breast cancer. Int J Cancer 2008;122:2562-2567.
13 Langer I, Guller U, Berclaz G, Koechli OR, Moch $\mathrm{H}$, et al.; Swiss Multicenter Study Group Sentinel Lymph Node in Breast Cancer: Accuracy of frozen section of sentinel lymph nodes: a prospective analysis of 659 breast cancer patients of the Swiss multicenter study. Breast Cancer Res Treat 2009;113: 129-136.

14 Schrenk P, Konstantiniuk P, Wölfl S, Bogner S, Haid A, Nemes C, Jagoutz-Herzlinger M, Redtenbacher S: Prediction of non-sentinel lymph node status in breast cancer with a micrometastatic sentinel node. Br J Surg 2005;92:707-713.

15 Gallowitsch HJ, Kraschl P, Igerc I, Hussein T, Kresnik E, Mikosch P, Kohlfuerst S, Hausegger K, Lind P: Sentinel node SPECT-CT in breast cancer. Can we expect any additional and clinically relevant information? Nuklearmedizin 2007;46:252-256.

16 Veronesi U, Galimberti V, Paganelli G, et al.: Axillary metastases in breast cancer patients with negative sentinel nodes: A follow-up of 3548 cases. Eur J Cancer 2009;45:1381-1388.

17 Konstantiniuk P, Schrenk P, Reitsamer R, Koeberle-Wuehrer R, Tausch C, Roka S, Riedl O, Poestlberger S, Hecke D, Janauer M, Haid A: A nonrandomized follow-up comparison between standard axillary node dissection and sentinel node biopsy in breast cancer. Breast 2007;16:520-526. 